In those cases where the symptoms which confront the practitioner are those of profound collapse, it becomes a question as to what course to pursue. Shall he operate at once at the risk of extinguishing the feeble spark of life, or shall he wait and endeavor to secure reaction, partial at least, before doing so? In my opinion, the latter course is the wiser. $\mathrm{He}$ should remember, however, that the shock is primarily due to the rupture and that the collapse is prolonged and deepened secondarily by the internal hemorrhage. As soon as there is some return of the pulse he should open the abdomen, seek for the sac, ligate close to the uterus and remove it, thereby stopping all further hemorrhage.

It was formerly advised to wash out the blood, insert a tube and drain. I believe this bad practice. In the first case I report, had I been content with simply sponging out the pelvis, accurately closing the wound, the uncovering of the wound would not have been followed by infection, and the patient would probably have recovered.

The practitioner should then sponge the abdomen as rapidly as possible with aseptic gauze, and having removed all that he can readily get rid of without prolonging the process, should close the abdomen without drainage.

The blood left is not septic and will be absorbed. and all the more readily, too, if the peritoneum be not disturbed by the flushing. If the patient has lost a great deal of blood and the abdomen is full of it, as in the second case $I$ have reported, no amount of flushing will remove it all, and precious time is lost in the attempt. All that can be readily removed by the hands and by sponging, should be quickly cleared ont, the wound closed and the patient put to bed. Hot bottles and strychnin, digitalis and whisky, should be resorted to hypodermically as may be necessary to sustain the heart's action and favor reaction.

It has been my fortune to meet with one case of abdominal pregnancy where the fetus had reached full term before its death. The case was brought to me in 1885 with what was supposed to be an ovarian tumor. I found a tumor which extended transversely from the left lumbar region to beyond the median line, and from the brim of the pelvis below to the margin of the ribs above. She had had it for fifteen years. Lately she had complained a great deal of colicky pains and troublesome constipation. The colicky pains had been so distressing that she had become addicted to the use of morphin to relieve them, and this in turn had promoted the constipation. The tumor had a very hard and smooth surface with somewhat irregular borders. Over the surface of the tumor there was dullness at some points, resonance at others. After explaining to her that it was very doubtful.if it could be removed, I proposed an exploratory incision, to which she assented. I found that the omentum and small intestines were adherent to it; in fact, it seemed imbedded in them. It presented a somewhat broad, flattened, smooth surface which looked like bone or cartilage. Suffice it to say that it was clearly unadvisable to attempt its removal. The wound was stitched up and healed by first intention. Great irritability of stomach persisted, her strength failed and she died two weeks after the operation. A postmortem examination was made and the bones of a fetal skull at full term was found, with traces of the legs and arms. In connection with the left ovary there seemed to be a piece of the bone of an arm.
Between this and the mass above there was no apparent connection whatever. Evidently there had been a tubal pregnancy of left side with rupture of the sac, the fetus' having survived the rupture and continued to develop to full term in the abdomen, when death ensued.

Two other cases have fallen under my care, both of which I believe to have been tubal pregnancy with rupture.

Mrs. H., aged about 35 years, mother of five children, had eaten a hearty dinner including corn. In the evening she suffered from considerable disturbance of the bowels. While in the water closet she was taken with violent pains in the abdomen, with great prostration, vomiting, etc. When called to see her the next morning, Sept. 9, 1895, I found her almost pulseless, cold, clammy skin, suppression of urine and great tenderness of abdomen. There was also some bloody discharge from the vagina. This condition deterred me from attempting an operation. She never rallied, and died on September 14, five days from the time of the attack. No autopsy.

Mrs. D., aged 26, mother of three children. Had one miscarriage between the first and second child. Had passed her menstrual period some four or five days when the flow commenced, which she regarded as delayed menstruation. This continued for four days. November 15, 1895, it stopped suddenly. She supposed she had taken cold, especially as she had some pain. On November 18 she was taken with severe pain in the left pelvis which "doubled her up," as she expressed it accompanied with some faintness and also diarrhea. I saw her in the afternoon. There was still pain, tenderness very great upon digital examination and upon suprapubic palpation, with quickened pulse, etc. I mentioned my suspicion of extrauterine pregnancy, and suggested laparotomy, which she emphatically declined. In the meantime there was a return of the bloody flow. She had some fever for a few days, temperature reaching $103 \mathrm{~F}$. and pulse 130 . The flow continued for about three weeks. She gradually got better. Some fullness and great tenderness in left pelvic region, and which has not yet, May 21, entirely disappeared.

That some accident happened in this case, I think, can not be questioned. The clinical history, symptoms and subsequent history point to extra-uterine pregnancy.

\section{MODERN METHODS OF TREATING THE MAXILLARY AND OTHER SINUSES OF THE CRANIUM.}

Read in the Section on Dentul and Oral Surgery, at the Forty seventh Annual Meeting of the American Medical Association beld at At]untu. Ga., May 5.8, 1896 .

BY W. XAVIER SUDDUTH, A.M., M.D.

FELLOW OF TFE CHICAGO ACADEMY OF MEDICINE, CHICAGO, ILL.

Pathology.-The diseases to which the sinuses of the head are liable are many. The most common, however, are empyema, tumors and necrosis of the walls from syphilis, mentioned in the order of their importance and frequency of occurrence.

Empyema antri is a suppurative condition and arises in the maxillary sinus, in a large proportion of cases from infection through dental abscesses, although the records show a considerable number of cases that can be traced to the extension of disease from the nasal cavity. In some instances the local inflammation undoubtedly arises from occlusion of the osteum maxillare by polypi or inflammation and decomposition of the retained secretion of the cavity walls. The most common cause of obstruction of the natural outlets of the antri is nasal polypi situated around the opening. The final result in any case is suppuration within the cavity of the antrum.

In chronic empyematous conditions of the antral cavity the lining membrane is generally found to be thickened and spongy in character, the caliber of the 
cavity in some cases is considerably reduced thereby. In not a few cases necrosis of the bony walls, results from the internal disease.

More or less intense neuralgias following the course of the nerve supply, together with sympathetic affections of the eye, resulting through the intimate nerve supply or caused by pressure of the inclosed pus upon the floor of the orbit, which also forms the roof of the antrum are found. These conditions are generally unilateral as empyema of the antrum is seldom bilateral.

Symptomatology.-The symptoms may be divided into two classes dependent upon whether there is occlusion of the osteum maxillare or not. In most cases it can also be said that the difference in symptomatology depends upon whether the disease arises from a dental or a nasal source, as occlusion of the osteum maxillare is most commonly, if not entirely, found accompanying the inroad of the disease from the nasal cavity.

Where obstruction of the natural outlet exists, there is a greater or less accumulation of matter within the cavity, with consequent pressure upon contiguous parts and more or less marked facial neuralgia. In some instances where the empyematous condition becomes chronic, necrosis of the osseous walls, most commonly on the nasal side, results and a copious discharge occurs into the nasal cavity. When the quantity of pus is large and does not find free outlet it may produce absorption of the orbital plate and infiltration into the tissues about the eye, in some cases producing glaucoma by involving the optic nerve.

In other instances the frontal sinus of the same side becomes involved as do also the sphenoidal sinuses, accompanied by marked supra-orbital neuralgia.

In empyema antri, without closure of the osteum maxillare, there is a more or less constant discharge of pus from the affected side, especially when patients lie upon the opposite side, a position seldom assumed because the discharge annoys them to such an extent they can not sleep. As a result of the constant discharge of pus into the throat, more or less interference of the general health follows. Dyspepsia and poisoning of the whole system results, which is indicated by a decided cachexia especially noticeable by the yellow appearance of the conjunctiva.

Diagnosis involves a differential consideration of the symptoms present in empyema of the several cavities of the head, including the diseases of the nasal fossa.

As pain is one of the constant symptoms, we use that as the first basis of comparison.

The diagnosis may also be established by exclusion, beginning with the maxillary sinus. Many different ways have been advocated to establish a diagnosis of empyema of the antrum. The simplest method is that of tapping with a curved hypodermic needle, through the nasal membrane, making the puncture in the inferior or middle meatus, and directing the point of the needle backward and downward. If there is pus present it may be accurately ascertained by such means.

Not every case of empyema antri should be opened, and great care should be used in making a diagnosis. Acute cases seldom come into our hands, by reason of the fact that nature restores the parts to a bearable if not a normal condition, and this fact should teach us a lesson reyarding surgical interference in acute cases.

Transillumination has been advocated by some, but has failed in the hands of even the most expert specialists when put into actual practice. I have used it in several cases but with indifferent success. In one case it was used before tapping and drawing off a considerable quantity of pus, and then again afterward, but no noticeable difference in the appearance could be observed.

The one remaining condition to be differentiated from suppuration of the antrum is feted ozena. Rhinoscopic examination will, in all cases, establish the diagnosis, as in the latter condition the disease of the mucous membrane will be readily observed, and, in all probability, found to be bilateral; while empyema antri is, in a large per cent. of cases unilateral.

The greatest difficulty in the diagnosis is found where complications arise by reason of several of the cavities being diseased at the same time. These cases do not come to the dental practitioner for treatment as often as they pass into the hands of the nose and throat specialist. Empyematous conditions of the antrum are generally of a purulent nature from the beginning, by reason of the fact that the cause generally lies in the discharge of a collection of pus from an alveolar abscess into the antrum.

Treatment of these cases is best directed from within the oral cavity, although the same methods may be adopted of introducing a tube through the nasal septum as before described. This is preferable to sacrificing a fairly good tooth or even trephining the outer plate in the region of the canine fossa. The latter should invariably be done, if the patient, for cosmetic reasons, objects to having the tube exposed upon the face, for if it is introduced through the outer plate it may be entirely concealed within the mouth. The method to be adopted is as follows: Select a trephine and a flexible rubber catheter of the same caliber, No. 7 or 8 being the size I generally use, and place them in an antiseptic solution.

Have the patient rinse the mouth thoroughly with a borated soda solution or listerin; dip the trephine in carbolic acid solution 5 per cent, or inject the tissue in the region where it is intended to insert the instrument with a 4 per cent. cocain solution and then make the opening and apply first dressing through this; then insert the tube, which should be ligated to a band or clasp that had previously been made and cemented to a conveniently located tooth. A short piece of tube or piping should be soldered to the band before putting it in place. The rubber tube should be about three or three and one-half inches in length, so as to enter the antrum from one-half to three-fourths of an inch and leave two inches outside. The free end should be kept plugged with a soft wooden pin, so as to prevent the saliva and air from entering, both of which may become a source of infection.

After placing the tube, the antrum should again be washed out thoroughly with a biborate of soda solution, the fluid to be introduced through the tube and allowed to flow out through the natural opening into the nasal cavity, the patient being instructed to incline the head forward. If the opening is found to be closed or too small to permit the fluid, on which the pus freely floats, to pass out, then a freer opening should be made. After the cavity has been thoroughly cleansed by the soda solution, a 3 per cent. pyrozone solution should be introduced and permitted sufficient time to disintegrate the remaining pus, if any, after which as a final dressing some bland antiseptic, such 
as a well diluted listerin, should be thrown into the cavity, and allowed to remain.

If the patient is told to lean forward when the washing out process is begun no part of the discharge will pass into the mouth or throat, a point that will be greatly appreciated. In like manner tubes may be inserted into all the cavities of the cranium and the cavities should be washed out two or three times a day. By aid of the tube this may be done by the patient or some member of the family and the case need not be seen by the practitioner more than twice or three times a week at the most.

If there is any odor at the second sitting, perman. ganate of potash should be substituted for the listerin, for a few days. As soon as the quantity of pus becomes lessened, then a stimulating antiseptic should be substituted. I do not know any that is better than sozo-jodol and its compounds. Sozo-iodol and bismuth in acute cases, sozo-iodol and mercury for sub-acute, and sozo-iodol and zinc for chronic cases. These should be used in the strength of about onehalf grain to the ounce of distilled water. All solutions should be warmed before using, including the $\mathrm{H}_{2} \mathrm{O}_{2}$, which may be done by placing the vessel containing the fluid in a dish of hot water.

If neuralgia follows the washing process, it is generally indicative that the astringent solution is too strong.

\section{A DISCUSSION ON ANATOMY OF THE ANTRUM OF HIGHMORE.}

Read in the Section on Dental and Oral Surgery, at the Forty-seventh Annual Meeting of the American Medical Association at

\section{BY M. H. FLETCHER, M.S., M.D., D.D.S.} CINCINNATI, OHIo.

The two following quotations are from the discussion on a paper by the present author, entitled "What a Dentist saw in Examining 500 Crania," read before the Mississippi Valley Dental Association, April 18, 1895.

Dr. J. E. Cravens; "I believe that if the Doctor had examined the skuils in the living subjects he would have found his proposition a mistake. I believe that nature never pushed the apex of a tooth root into the Highmorian cavity, without a bony protection, however thin. She never completed the apex of the root of a tooth in the mucous membrane alone."

Dr. H. A. Smith: "I doubt very much the entire absence of bone. If the position taken by Dr. Fletcher is true it may account for certain lesions, phases of facial neuralgia. I doubt very much if the root of a tooth ever penetrates the antrum. You must have a thin covering of bone. The bone of the antrum is proliferated by its own periosteum, and does not depend on any tissue of the teeth. If you take those skulls and examine the roots of the teeth you assume that they never had a covering of bone, but they may have had, even though it was as thin as paper. If you examine those skulls, where the bone is denuded it is so thin that in the dry state it falls away. An observation made on a dry skull is not conclusive. If it is true that the roots will penetrate the antrum it will account for a great many of those cases of facial neuralgia."

These criticisms were brought out by the following statement made in the paper referred to:

Dr. M. H. Fletcher: "As to diseases of the antrum, it seems to me that these people were remarkably

exempt, when we consider that they could have had no surgical or medical attention, and also that about 25 per cent. of them had abscessed upper molars. This fact is significant when it is claimed by the majority of authors that diseases of the antrum come more frequently from this class of teeth than from any other source. This series of examinations show that out of the 252 cases of abscessed upper molars, only 12 perforated the antrum. This would seem a remarkably small number and indicate that abscessed teeth do not cause antral trouble as often in comparison with other causes of inflammation of this cavity as most authors maintain they do."

My reasons for believing that the teeth may often be affected by diseases of the antrum are strengthened by considering a certain feature of the anatomy of the parts, mentioned by Gray, and omitted by most other anatomists, that, "in some cases the floor is perforated by the teeth in this situation." I found this to be the condition in twenty cases, showing that about 4 per cent. of persons have, normally, nothing covering the apices of these teeth but mucous membrane (I wish to say that the statistics on this particular point are not accurate, on account of the inability to see into the antrum, in an unbroken skull). Many skulls were broken, however, so that the examinations could be thoroughly made on this point, as well as others, and when they were not broken the sense of touch was used to determine the presence of bony processes, septa and the general form of the cavity, and the normal openings above the teeth, as far as possible. It was also observed that these normal openings occurred where the floor of the antrum was comparatively fiat, and not where there was a conical process, and that these cases where the conical processes occurred were almost invariably covered with a considerable thickness of bone.

These conditions being present, it would seem a natural result when the nucous membrane of the floor of the antrum becomes broken down, for the blood and nerve supply of teeth (so perforating the floor) to be interfered with, and possibly entirely destroyed, since the apical foramen of the teeth must be exposed to these destructive influences. In cases of occlusion of the osteum maxillare and other openings into the nose, the antrum may become tensely engorged, and under this condition if there be no bony covering to the apex of the teeth, it would be more or less driven from its socket and become very sore to the touch.

It is my belief that if accurate statistics could be had they would indicate that the exciting causes of diseases of the antrum is ten to one in favor of intranasal disorders. I make this statement, taking into consideration the large per cent. of teeth that are known to perforate the bony floor of the antrum, for my observations also show that a much larger num. ber of teeth are denuded of bone on the buccal surface of the alveolus, hence, the large preponderance of abscessed teeth which discharge in this locality, a place where one familiar with the disease invariably looks for a fistulous opening. In this situation, if the bone is not entirely absent in some spot, it is so thin that it offers to internal pressure the least possible resistance of any other part of the sockets of the teeth.

During the discussion of the paper referred to I corrected the statement that "These cases have, normally, nothing covering the apieces of the teeth, but mucous membrane." The words mucous mem- 